\title{
BEHAVIOR OF SURFACTANT MIXTURES AT SOLID/LIQUID AND OIL/LIQUID INTERFACES IN CHEMICAL FLOODING SYSTEMS
}

\section{SEMIANNUAL TECHNICAL PROGRESS REPORT}

Reporting Period Start Date: 03/01/2002

Reporting Period End Date: $\quad$ 09/01/2002

Principle Author: $\quad$ Prof. P. Somasundaran

Date Report Issued: $\quad$ Sept. 30, 2002

DOE Award Number: $\quad$ DE-FC26-01BC15312

Principal Prof. P. Somasundaran

Investigator

Submitting Organization Office of Projects and Grants

Columbia University in the City of New York

1210 Amsterdam Avenue, Mail Code 2205

Room 254 Engineering Terrace

New York, NY 10027

Contracting Officer's

H.V. Weyland

Representative

U.S. Department of Energy,

Bartlesville Project Office,

P.O. Box 1398,

Bartlesville, OK 74005. 


\section{DISCLAIMER}

This report was prepared as an account of work sponsored by an agency of the United States Government. Neither the United States Government nor any agency thereof, or any of their employees, makes any warranty, express or implied, or assumes any legal liability or responsibility for the accuracy, completeness, or usefulness of any information, apparatus, product, or process disclosed, or represents that its use would not infringe privately owned rights. Reference herein to any specific commercial product, process, or service by trade name, trademark, manufacturer, or otherwise does not necessarily constitute or imply its endorsement, recommendation, or favoring by the United States Government or any agency thereof. The views and opinions of authors expressed herein do not necessarily state or reflect those of the United States Government or any agency thereof. 


\begin{abstract}
The aim of the project is to develop and evaluate efficient novel surfactant mixtures for enhanced oil recovery. Surfactant loss by adsorption or precipitation depends to a great extent on the type of surfactant complexes and aggregates formed. Such information as well as techniques to generate the information is lacking currently particularly for surfactant mixtures and surfactant/polymer systems. A novel analytical centrifuge application is explored during the last period to generate information on structures-performance relationship for different surfactant aggregates in solution and, in turn, at interfaces. To use analytical untracentrifuge for surfactant mixtures, information on partial specific volumes of single surfactants and their mixtures is required. Towards this purpose, surface tension and density measurements were performed to determine critical micellar concentrations ( $\mathrm{cmc}$ ), partial specific volumes of n-dodecyl-\$-Dmaltoside (DM), nonyl phenol ethoxylated decyl ether (NP-10) and their $1: 1$ mixtures at $25^{\circ} \mathrm{C}$. Durchschlag's theoretical calculation method was adopted to calculate the partial specific volumes. Effects of temperature and mixing, as well as methods used for estimation on micellization and partial specific volumes were studied during the current period.

Surface tension results revealed no interaction between the two surfactants in mixed micelles. Partial specific volume measurements also indicated no interaction in mixed micelles. Maximum adsorption density, area per molecule and free energy of micellization were also calculated. Partial specific volumes were estimated by two experimental methods: $d D d d c$ and $V_{F}$. The difference between the results of using the two methods is within $0.5 \%$ deviation. It was found that the partial specific volume is concentration dependent and sensitive to changes in temperature. The information generated in this study will be used for the study of surfactant aggregate mass distribution in mixed systems. Such information will in future be used to identify optimum surfactant systems.
\end{abstract}




\section{TABLE OF CONTENTS}

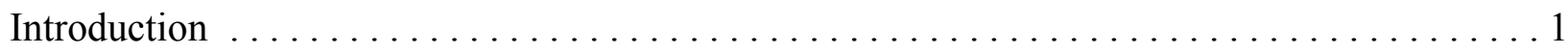

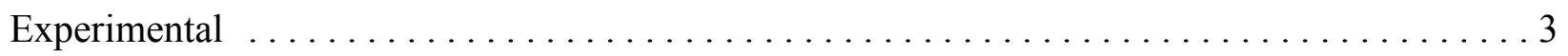

Results and Discussions $\ldots \ldots \ldots \ldots \ldots \ldots \ldots \ldots \ldots \ldots \ldots \ldots \ldots \ldots$

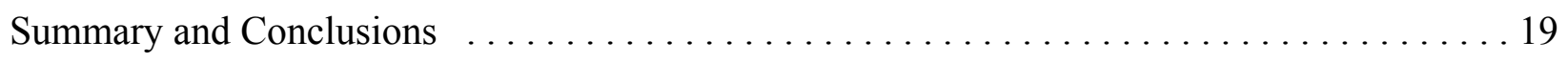

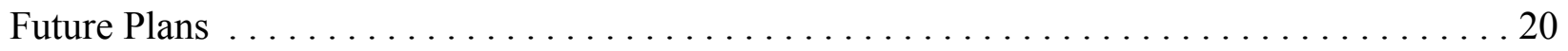

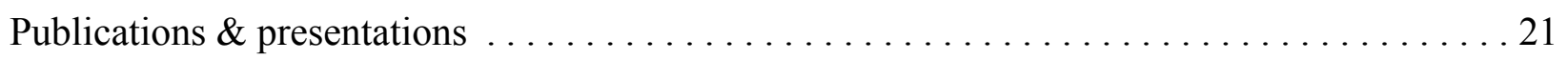

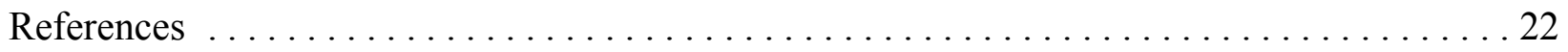




\section{LIST OF GRAPHICAL MATERIALS}

Figure 1. Surface tension of n-dodecyl-\$-D-maltoside (DM) and nonyl phenol ethoxylated decyl ether (NP-10) and their 1:1 molar ratio mixtures.

Figure 2. Densities of n-dodecyl-\$-D-maltoside (DM) and nonyl phenol ethoxylated decyl ether (NP-10) and their 1:1 mixture as a function of volumetric concentrations.

Figure 3. Partial specific volume by $\mathrm{V}_{\mathrm{F}}$ (apparent molar volume) method versus times $\mathrm{CMC}$ of DM, NP-10 and their mixtures at $25^{\circ} \mathrm{C}$.

Figure 4. The molecular structures of n-dodecyl-\$-D-maltoside (DM) and nonyl phenol ethoxylated decyl ether (NP-10) .

Figure 5. Density of 1:1 mixture of n-dodecyl-\$-D-maltoside (DM) and nonyl phenol ethoxylated decyl ether (NP-10) aqueous solutions at different temperatures.

Figure 6. Partial specific volume of 1:1 mixtures at different temperature versus molarity.

Figure 7. Partial specific volumes versus temperature by $d D / d c \& V_{\Phi}$ methods. 


\section{INTRODUCTION}

The goal of the proposed research is to help develop improved extraction processes that can mobilize some of the oil left untapped using conventional techniques. It is our aim to design and evaluate efficient novel mixtures of surfactants for enhanced oil recovery, especially in multicomponents systems containing oil, polymers, emulsifiers and/or inorganics that are invariably present in the system. The key criterion for the successful application of the techniques using candidate surfactants is minimal loss of surfactants by adsorption and precipitation.

Our previous work has shown that aggregation behavior of some surfactant mixtures is quite unusual and more than one type of mixed micelles can form and possibly co-exist in mixed systems. This finding has both theoretical and practical implications. It has potential for applications in EOR to minimize interfacial tension between oil and the flooding media to facilitate oil liberation and, at the same time, to reduce the adsorption of surfactants on reservoir rocks.

A major hurdle in the analysis of mixed surfactant systems is the lack of information on the type of complexes and aggregates formed between various components and the lack of techniques for deriving such information. We have developed Analytical ultracentrifuge technique for the first time to study the relationship between distribution of surfactant species and aggregates and performance of the surfactants and their mixtures. Both dynamic and equilibrium information, such as the weight, shape and types of micelles, can be obtained with analytical ultracentrifuge from sedimentation velocity and equilibrium experiments. To get this information, partial specific volume of surfactants needs to be obtained as one of the fundamental parameter.

During the last reporting period, mineral samples, surfactants and other chemicals to be used 
in this project have been procured and characterized, protocol for analytical centrifugation established and initial analytical ultracentrifuge tests conducted to identify the best analytical method, calculate the partial specific volume and collect information on single surfactant systems. The partial specific volume of NP-10 is calculated by Durchschlag's theoretical calculation method. Three software, Optima ${ }^{\mathrm{TM}}$ XL-A/XL-I data analysis software, DCDT+ and Svedberg were compared for sedimentation velocity data analysis. The sedimentation coefficient and aggregation Number of NP-10 are obtained from the above three software and Svedberg was found the best.

During this period, surface tension and density measurements were carried out to determine critical micellar concentrations (cmc), partial specific volumes of n-dodecyl-\$-D-maltoside (DM) and nonyl phenol ethoxylated decyl ether (NP-10) and their 1:1 mixtures at $25^{\circ} \mathrm{C}$. Durchschlag's theoretical calculation method was adopted also to calculate partial specific volumes and compared with the above two methods. Based on surface tension data, the cmc, interaction parameters of the surfactants in mixed micelles, maximum adsorption density, area per molecule and free energy of micellization were calculated. Effects of methods used for the determination, temperature, mixing and micellization, on partial specific volumes are discussed here. 


\section{EXPERIMENTAL}

\section{Materials:}

Nonionic surfactants, n-dodecyl-\$-D-maltoside (DM) of greater than 95\% purity and nonyl phenol ethoxylated decyl ether (NP-10) of 99\% purity, were obtained from Calbiochem and Nikko Chemicals, respectively. They were used as received. Triple distilled water was used to prepare all the solutions and all the experiments were performed with freshly prepared solutions.

\section{Methods:}

Surface tension measurements of n-dodecyl-\$-D-maltoside (DM) and nonyl phenol ethoxylated decyl ether (NP-10) and their mixtures were made by the Wilhelmy plate method with correction factors. The pull exerted on the plate was determined by a Cahn microbalance (Model LM 600). The entire assembly was kept in a draft-free plastic cage at $25 \pm 0.2{ }^{\circ} \mathrm{C}$. Before each measurement, the plate was burnt to red and then cooled down to remove residual organics. The plate was in contact with the surfactant solution for 30 minutes to allow equilibrium prior to the measurement. Surface tension of triple distilled water was measured at $25^{\circ} \mathrm{C}$ prior to each set of experiments.

Densities of surfactant solutions were measured with an Anton Paar DMA 5000 density meter. The principle is to measure the period of oscillation of a U-shape tube with the sample inside. Operation temperatures were set at $25,30,36.8,40,45$ and $50^{\circ} \mathrm{C}$. The accuracy of the density and temperature were $5^{*} 10^{-6} \mathrm{~g} / \mathrm{cm}^{3}$ and 0.01 , respectively. The instrument was calibrated at atmospheric pressure (1013 mbar) with air and Anton Paar's standard water $\left(0.998201\right.$ and $0.000010 \mathrm{~g} / \mathrm{cm}^{3}$ at $20^{\circ} \mathrm{C}$ ). Acetone was used to rinse the U-tube between measurements and was dried by filtered air. 


\section{RESULTS AND DISCUSSIONS}

\section{Surface tension measurements and related parameters}

Surface tension of n-dodecyl-\$-D-maltoside(DM) with Nonyl phenol ethoxylated decyl ether (NP-10) and their 1:1 molar ratio mixtures was measured at $\mathrm{pH} 6.5$ and $25^{\circ} \mathrm{C}$ and data were shown in Figure 1 as a function of concentration.

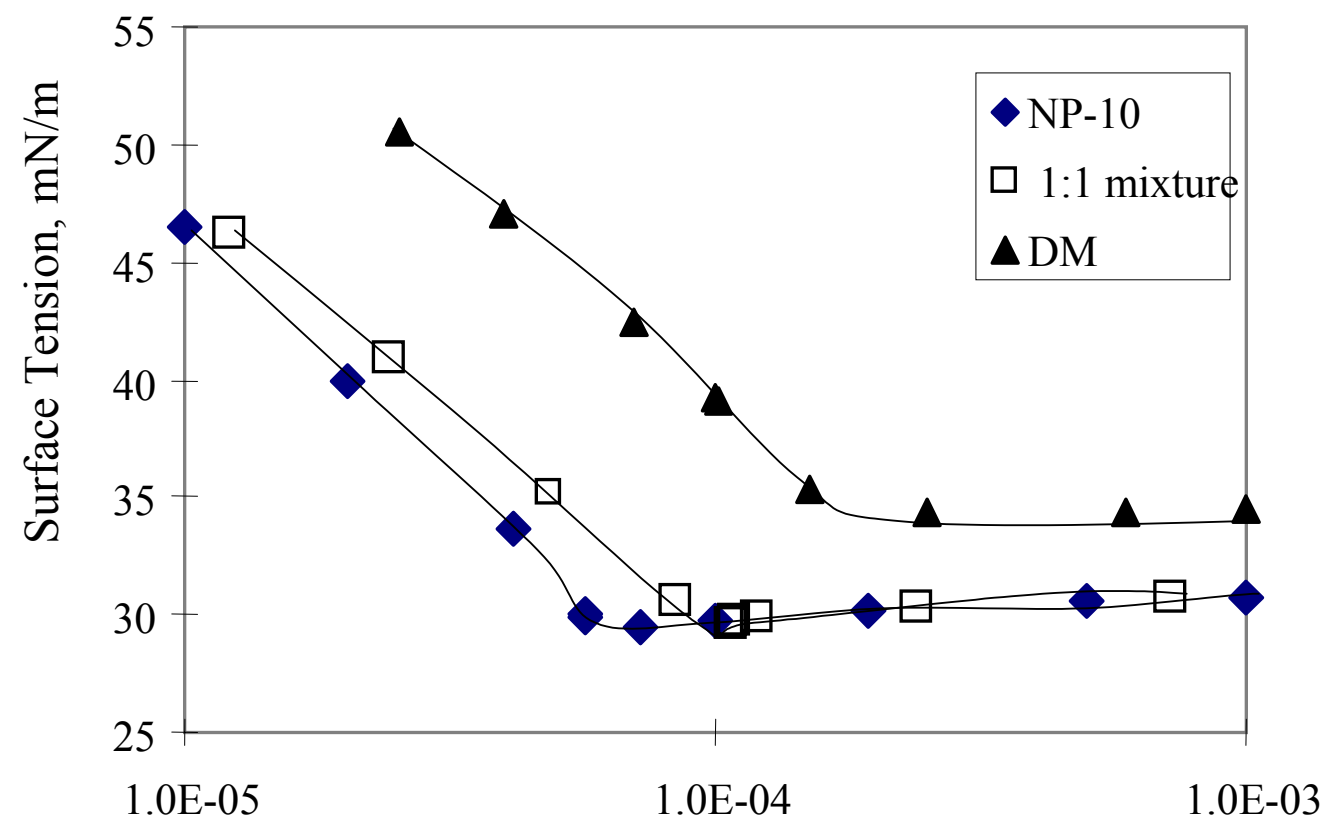

Concentration, $\mathrm{M}$

Figure 1. Surface tension of n-dodecyl-\$-D-maltoside (DM) and Nonyl phenol ethoxylated decyl ether (NP-10) and their 1:1 molar ratio mixtures.

Relevant data such as the critical micelle concentrations $(\mathrm{cmc})$, the mole fraction of DM in the monolayer and in the mixed micelles, and the interaction parameters $\$$ for the mixtures in micelles and at air/water interface, maximum adsorption ' ${ }_{\max }$, area per molecule $A_{\min }$ and free energy of micellization ) $\mathrm{G}_{\text {mic }}$ are listed in Table 1 and 2 . 
$\mathrm{Cmc}$ is obtained from the intersection before and after $\mathrm{cmc}$ in surface tension curve. The strength of interaction between these two surfactants was determined by the interaction parameter $\$$. Negative interaction parameter corresponds to synergism while positive interaction parameter indicates antagonism.

Table 1: parameters obtained from surface tension measurement

\begin{tabular}{|r|c|c|c|c|c|}
\hline & $\begin{array}{c}\mathrm{cmc}^{*} 10^{4} \\
(\mathrm{M})\end{array}$ & $\begin{array}{c}\left(_{\min }\right. \\
(\mathrm{mN} / \mathrm{m})\end{array}$ & $\begin{array}{c}\mathrm{max}^{*} 10^{6} \\
\left(\mathrm{~mol} / \mathrm{m}^{2}\right)\end{array}$ & $\begin{array}{c}\mathrm{A}_{\min } \\
\left(\mathrm{D}^{2}\right)\end{array}$ & $\begin{array}{c}\mathrm{G}_{\text {mic }} \\
(\mathrm{kJ} / \mathrm{mol})\end{array}$ \\
\hline DM & 1.8 & 34.3 & 3.37 & 49.3 & -31.3 \\
\hline DM (ref. 1) & 1.5 & 36.23 & 3.32 & 49.9 & -31.8 \\
\hline Mixtures & 0.96 & 29.6 & 3.28 & 50.7 & -32.9 \\
\hline NP-10 & 0.62 & 29.5 & 3.78 & 43.9 & -34.0 \\
\hline
\end{tabular}

Table 2: Interaction parameter \$ at air/water interface and in micelles, and mole fraction of DM in the monolayer and the micelles at $\mathrm{cmc}$

\begin{tabular}{|l|r|}
\hline interaction parameter at the air/water interface & -1.23 \\
\hline$X$ of DM at the air/water interface & 0.28 \\
\hline interaction parameter in the micelle & 0.22 \\
\hline$X$ of DM in water & 0.24 \\
\hline
\end{tabular}

From surface tension data, it is seen that NP-10 is more surface active than DM and the mixed micelle is NP-10 dominant at cmc. Our data are very close to the results reported in the literature [1]. The interaction parameter between DM and NP-10 in the mixed micelle is close to zero $(\$=0.22)$. This suggests that there is no interaction between these two molecules in the micelles and that the system is ideal. This phenomenon is normal for nonionic surfactant interaction. It has been reported in our previous investigation that the interaction between DM and C12EO5 is ideal. At the air water interface, the hydrophilic group stays in water and the hydrophobic group stretches out to air. The stronger interaction at the air/water interface may result from the hydrogen bonding 
of EO groups (which is the hydrophilic group of NP-10 surfactant and points toward the water upon forming monolayer) and the sugar group (which is the hydrophilic group of DM and anchors inside the water with the carbon chain reaching away from the water surface) with water molecules. On the other hand, the phenol group in NP-10 molecule is directed towards air and facilitates the formation of monolayers.

Data for cmcs of DM, NP-10 and their mixtures were used to calculate concentration normalized to $\mathrm{cmc}$ for partial specific volume determination and estimation.

\section{Partial specific volumes of DM, NP-10 and 1:1 mixtures by density experiments}

By definition, partial specific volume is the volumeric change upon adding one gram of material to water. Its unit is $\mathrm{cm}^{3} \mathrm{~g}^{-1}$. The partial specific volume is obtained experimentally using two methods. The first one is used more frequently [2]:

$$
\bar{v}=\frac{1}{\rho_{0}}\left(1-\frac{d \rho}{d C}\right)
$$

c is surfactant volumeric concentration in $\mathrm{g} / \mathrm{ml}$. Dand $\mathrm{D}_{6}$ are the densities of the solution and solvent, respectively.

The second method calculated apparent molar volume first [3]:

$$
\mathrm{V}_{\mathrm{F}}=\mathrm{M} / \mathrm{D} \cdot 10^{3}(\mathrm{D} \cdot \mathrm{D}) /(\mathrm{mDD})
$$

then the partial molar volume $\bar{V}_{C}$

$$
\bar{V}_{\bar{C}}^{=} \mathrm{V}_{\mathrm{F}}+\mathrm{m}(\mathrm{M} / \mathrm{Mm})_{\mathrm{T}, \mathrm{P}}
$$

$\mathrm{V}_{\mathrm{F}}$ is the apparent molar volume, $\bar{V}_{C}$ is the partial molar volume, $\mathrm{M}$ is molecular weight of the solute, and $\mathrm{m}$ is molarity.

Finally, partial specific volume is obtained from:

$$
\bar{v}=\overline{V_{C}} / M
$$


The partial specific volumes of DM, NP-10, their $1: 1$ mixtures at $25^{\circ} \mathrm{C}$ are determined from data from density measurement using the above equations.

Figure 2 shows their densities as a function of logarithm of volumeric concentration. The slope of density over concentration decreases in the order of DM, 1:1 mixture and NP-10.

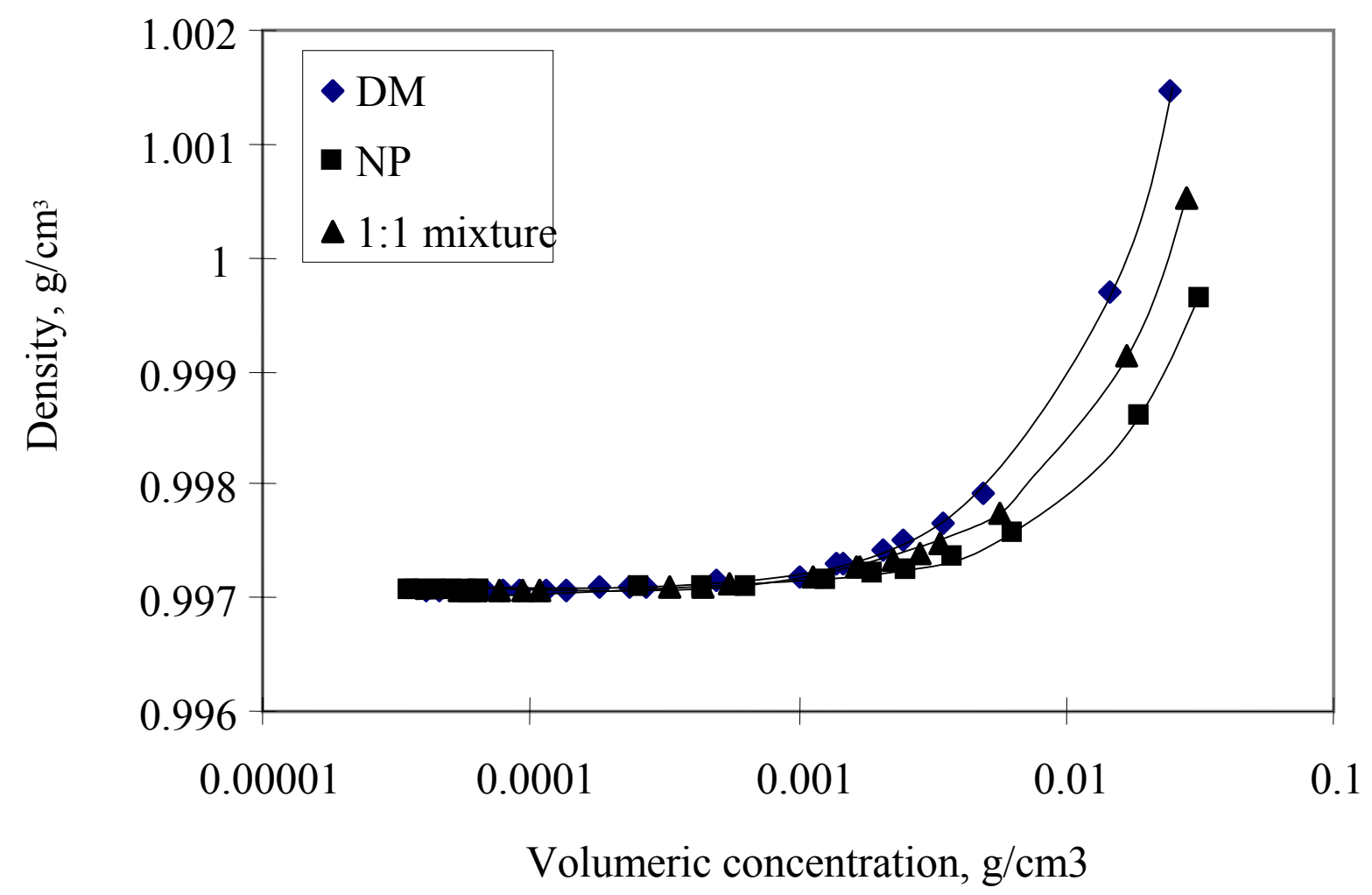

Figure 2. Density of n-dodecyl-\$-D-maltoside (DM) and Nonyl phenol ethoxylated decyl ether (NP-10) and their 1:1 mixtures as a function of logarithm of volumetric concentrations.

The data were analyzed with dDdc method first. The partial specific volumes were calculated using equation (1) and classified into four categories with respect to cmc, i.e., $<1,1-10$, $10-50$ and $>100$ times cmc. 
Table 3: partial specific volumes of n-dodecyl-\$-D-maltoside (DM) and Nonyl phenol ethoxylated decyl ether (NP-10) and their 1:1 mixtures in different concentration ranges.

\begin{tabular}{|c|c|c|c|}
\hline \multirow{2}{*}{ Times cmc } & \multicolumn{3}{|c|}{ Partial specific volume by dD/dc method } \\
& \multicolumn{3}{|c|}{$\left(\mathrm{cm}^{3} \mathrm{~g}^{-1}\right)$} \\
\cline { 2 - 4 } & DM & $1: 1$ mixtures & NP-10 \\
\hline$<1$ & 0.477 & & 0.245 \\
\hline $1-10$ & 0.821 & 0.864 & 0.899 \\
\hline $10-50$ & 0.824 & 0.881 & 0.920 \\
\hline$>50$ & 0.820 & 0.879 & 0.922 \\
\hline
\end{tabular}

It is obvious that the partial specific volumes are the lowest at concentration below $\mathrm{cmc}$. It should be noted that the density of the surfactant solutions does not change with increase in concentration unless the concentration is in the close vicinity of $\mathrm{cmc}$. All the surfactant molecules are evenly distributed in solution at concentrations far below $\mathrm{cmc}$ and density of the solutions is the same as that of water. The partial specific volume far below $\mathrm{cmc}$ should be higher than 1.0 since the $\mathrm{dD} d \mathrm{dc}$ is zero and the partial specific volume is calculated using $1 / \mathrm{D}_{\mathrm{b}}$ which is 0.997043 at temperature of $25^{\circ} \mathrm{C}$. When the concentration is high enough to form micelles in the solution, the self-organized molecular aggregates have a water-free core containing dissolved oxygen. Evidently, micelles do contributes to the sharp increase in density. The current results show that the micelles form in solution at concentrations lower than at which monolayer forms at air/water interface.

The partial specific volumes increase in the range of 1-10 times cme to $10-50$ times cmc. This suggests possible change in packing format in mixed micelles. The differences in partial specific volumes from 1-10 times cme to 10-50 times cmc are 0.003 for DM, 0.017 for 1:1 mixture and 0.021 for NP-10 respectively, which corresponds to . The differences in numbers reveal that the change of micellar shape would follow the order: DM $<1: 1$ mixture $<$ NP-10.

The partial specific volume at concentrations above 50 times $\mathrm{cmc}$ does not change 
appreciably from that between 10 to $50 \mathrm{cmc}$. Thus a conclusion could be drawn that partial specific volume is not a constant for the same surfactant solution. Micellization has a significant effect on the partial specific volume for nonionic surfactants and their mixtures. The partial specific volume would be constant only when the concentration is higher than 10 times cmc.

When analyzing the data with $\mathrm{V}_{\mathrm{F}}$ (apparent molar volume) method, similar trends were obtained as seen in figure 3 .

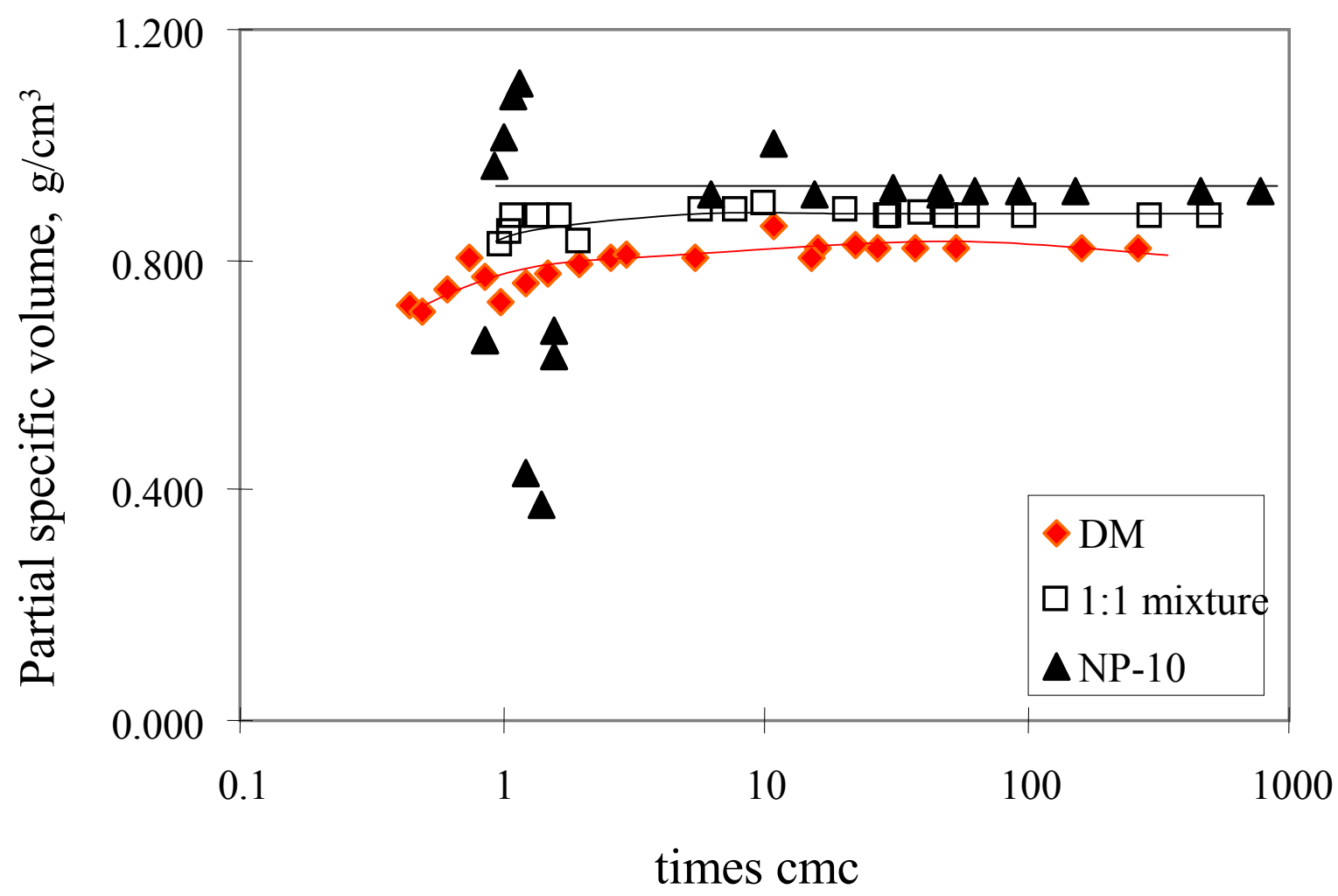

Figure 3. Partial specific volume by $\mathrm{V}_{\mathrm{F}}$ (apparent molar volume) method versus times cmc of n-dodecyl-\$-D-maltoside (DM) and Nonyl phenol ethoxylated decyl ether (NP-10) and their mixtures at $25{ }^{\circ} \mathrm{C}$.

These three curves exhibit the same trend showing partial specific volumes are constant at 
the concentrations above 10 times $\mathrm{cmc}$, whereas below $\mathrm{cmc}$, only data for partial specific volume of NP-10 shows scattering. NP-10 has good hydrophilicity and surface activity so that there is very little difference between water and NP-10 in density. This accounts for the scattering of the measured data at low concentrations. When the concentration is between 1-10 times $\mathrm{cmc}$, the partial specific volumes of DM and 1:1 mixture increase with concentration and reach maxima around 10 times cmc. This may correspond to transition of the packing format of surfactant molecules in mixed micelles due to change in concentration. Partial specific volumes of NP-10 in the same range, scattering at concentration near $\mathrm{cmc}$ and become less variant above 6 times $\mathrm{cmc}$. This phenomenon does not exist for the anionic and cationic surfactants with similar hydrocarbon chain length because cmcs of all these three compounds are very low. $\left(6 \times 10^{-6}-1.8 \times 10^{-4} \mathrm{M}\right)$. NP-10 has the highest partial specific volumes among all the three compounds. Thus the difference between densities from low to high concentration is relatively small. This is supported by the fact that the densities of NP-10 solutions are the same as that of water over a wide concentration range. The fluctuation of partial specific volume at different concentration is shown in table 4.

Table 4: partial specific volume range of n-dodecyl-\$-D-maltoside (DM) and Nonyl phenol ethoxylated decyl ether (NP-10) and their 1:1 mixtures

by apparent molar volume method

\begin{tabular}{|c|c|c|c|}
\hline Times cmc & \multicolumn{3}{|c|}{ Partial specific volume range by $\mathrm{V}_{\mathrm{F}}$ method } \\
\hline & $\mathrm{DM}$ & $1: 1$ mixture & $\mathrm{NP}-10$ \\
\hline$<1$ (range) & $0.71-0.80$ & 0.824 & $0.660-0.962$ \\
\hline $1-10$ (range) & $0.761-0.810$ & $0.830-0.897$ & $0.375-1.105$ \\
\hline $10-50$ (range) & $0.806-0.856$ & $0.873-0.885$ & $0.913-0.999$ \\
\hline$>50$ & $0.818-0.821$ & $0.876-0.878$ & $0.919-0.920$ \\
\hline
\end{tabular}

The partial specific volumes in each concentration range are averaged excluding maximum and minimum. The comparison of partial specific volumes derived from $V_{F}$ method are made and 
results are listed in table 5.

Table 5. Comparison of partial specific volumes using $V_{F}$ method with those using the dD'dc method

\begin{tabular}{|c|c|c|c|c|c|c|c|c|c|}
\hline \multirow{2}{*}{$\begin{array}{c}\text { Times } \\
\mathrm{cmc}\end{array}$} & \multicolumn{3}{|c|}{$\mathrm{DM}$} & \multicolumn{3}{c|}{$1: 1$ mixture } & \multicolumn{3}{c|}{$\mathrm{NP}-10$} \\
\cline { 2 - 11 } & $\mathrm{dD} d \mathrm{dc}$ & $\mathrm{V}_{\mathrm{F}}$ & )$<\%$ & $\mathrm{dD} d \mathrm{dc}$ & $\mathrm{V}_{\mathrm{F}}$ & $\%$ & $\mathrm{dD} d \mathrm{dc}$ & $\mathrm{V}_{\mathrm{F}}$ & )$<\%$ \\
\hline$<1$ & 0.477 & & & & & & 0.245 & & \\
\hline $1-10$ & 0.821 & 0.802 & 2.3 & 0.864 & 0.879 & -1.7 & 0.899 & & \\
\hline $10-50$ & 0.824 & 0.821 & 0.4 & 0.881 & 0.878 & 0.3 & 0.920 & 0.919 & 0.1 \\
\hline$>50$ & 0.820 & 0.820 & 0.0 & 0.879 & 0.877 & 0.2 & 0.922 & 0.920 & 0.2 \\
\hline
\end{tabular}

Here the $\mathrm{V}_{\mathrm{F}}$ denotes the apparent molar volume method. The comparison shows that these two methods agree with each other very well at concentrations above 10 times cmc. The agreement manifests that both methods are accurate for determination of partial specific volume. The values from both methods at concentration above 50 times $\mathrm{cmc}$ are averaged. The partial specific volumes show the tendency to increase in the order DM $<$ mixture $<\mathrm{NP}-10$. The partial specific volume is a function of the chemical structure, the temperature and micellization. If we examine their chemical structure, n-dodecyl-\$-D-maltoside (DM) has 12-carbon chain as hydrophobic tail and 2-sugar groups as hydrophilic head. SANS experiment showed that n-dodecyl-\$-D-maltoside (DM) micelle is oblate ellipsoid with $a=3.44$ and $b=2.03$ [4]. Nonyl phenol ethoxylated decyl ether (NP-10) has 9-carbon chain and 1 phenol group as hydrophobic part and 10 EO groups as hydrophilic part. Nonyl phenol ethoxylated decyl ether (NP-10) micelle is more asymmetrical with $\mathrm{a}=5.4$ and $\mathrm{b}=2.8$ according to the literature [5]. The bulky hydrophilic head of DM produces very little space between surfactant molecules in the micelles. On contrast, the linear NP-10 structure allows more water molecules containing in the micelles. Therefore, the partial specific volume of NP-10 is close to the that of water. In addition to molecular structure, strong hydration of NP-10 molecules contributes to the large value in partial specific volume. 1:1 mixture behaves as expected: the partial specific volume is larger than that of DM and smaller than that of NP-10. 
III. Partial specific volumes of DM, NP-10 and their 1:1 mixtures by Durchschlag's calculation method

Partial specific volume could also be theoretically calculated. Method of calculation is fast and accurate for single component systems and systems with inorganic salts.

Helmut Durchschlag and Peter Zipper's approach [6,7] was adopted by us to calculate the partial specific volumes of surfactants and their mixtures in aqueous solutions. The method is based on Traube's additivity principle and concept of volume increments for atoms. This calculation procedure is developed for increments/decrements for covolume, ring formation and ionization.. Partial molar volumes, $\bar{V}_{C}$ is defined as following:

$$
\overline{V_{C}}=\sum V_{i}+V_{C V}-\sum V_{R F}-\sum V_{E S}
$$

Where $V_{i}$ is the volume increment for any atom or atomic group, $V_{C V}$ is the correction due to the covolume, $V_{R F}$ and $V_{E S}$ stand for the decrease in volume caused by ring formation and ionization (electrostriction), respectively.

Figure 4. shows the molecular structures of DM and NP-10

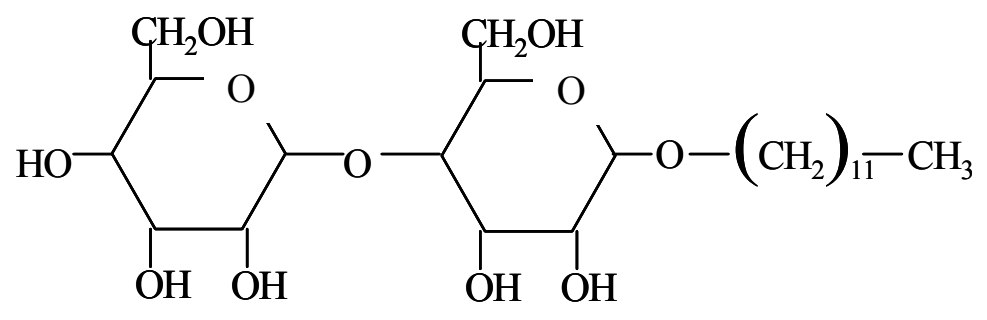

$$
\begin{aligned}
& \mathrm{DM} \quad \mathrm{C}_{24} \mathrm{H}_{46} \mathrm{O}_{11} \quad \mathrm{M}=510.6 \\
& \bar{V}_{C}=24 \times 9.9(\mathrm{C})+46 \times 3.1(\mathrm{H})+4 \times 5.5(\mathrm{O})+2 \times 2.3(\mathrm{O})+5 \times 0.4(\mathrm{O})+12.4\left(\mathrm{~V}_{\mathrm{CV}}\right)-2 \times 8.1\left(\mathrm{~V}_{\mathrm{RF}}\right)=405 \mathrm{~cm}^{3} / \mathrm{mol} \\
& \bar{v}=0.793 \mathrm{~cm}^{3} / \mathrm{g}
\end{aligned}
$$




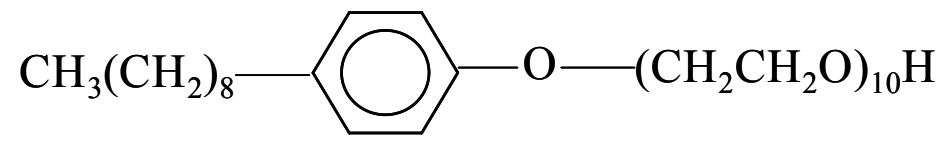

$$
\text { NP-10 } \quad \mathrm{C}_{35} \mathrm{H}_{64} \mathrm{O}_{11} \quad \mathrm{M}=660.9
$$

$$
\begin{aligned}
\bar{V}_{C} & =35 \times 9.9(\mathrm{C})+64 \times 3.1(\mathrm{H})+10 \times 5.5(\mathrm{O})+1 \times 2.3(\mathrm{O})+12.4\left(\mathrm{~V}_{\mathrm{CV}}\right)-8.1\left(\mathrm{~V}_{\mathrm{RF}}\right)=606.5 \mathrm{~m}^{3} / \mathrm{mol} \\
\bar{v} & =0.918 \mathrm{~cm}^{3} / \mathrm{g}
\end{aligned}
$$

As for the mixture, a simple method is used for the estimating:

$$
\bar{v}=\frac{\Sigma x_{i} \bar{V}_{C, i}}{\Sigma x_{i} M_{i}}
$$

$\bar{V}_{C, i}$ is the sum of partial molar volume of each component, $\Sigma M_{i}$ the sum of molecular weights and $x_{i}$ the molar ratio of one component over the other. For 1:1 DM-NP-10 mixture, the partial specific volume is 0.867 . The comparison of dDdc, $\mathrm{V}_{\mathrm{F}}$ and Durchschlag's methods are made in table 8. Data from the literature is also tabulated below.

Table 8. List of partial specific volumes and deviation for DM, NP-10 and their 1:1 mixture by empirical and theoretical methods.

\begin{tabular}{|c|c|c|c|}
\hline Methods & DM & $1: 1$ mixture & NP-10 \\
\hline $\mathrm{dD}$ dc & 0.820 & 0.879 & 0.922 \\
\hline $\mathrm{V}_{\mathrm{F}}$ & 0.820 & 0.877 & 0.920 \\
\hline Durchschlag's & 0.793 & 0.867 & 0.918 \\
\hline < $\%$ \% & -3.3 & -1.3 & -0.3 \\
\hline literature value & $0.837[6]$ & & $0.922[7]$ \\
\hline
\end{tabular}

The averages of partial specific volumes by $d D d c \& V_{F}$ methods are compared with that by Durchschlag's method. The deviations show that the difference is decreasing following the order of DM $>1: 1$ mixture $>$ NP-10. The large difference of DM results from the structure. The hydrophilic group of DM is composed of two sugar rings connected by an oxygen atom to form \$linkage. The connection of two sugar rings is rigid and can not be bent. It can be seen that the bulky sugar groups not only bring deviation to the partial specific volume of DM, but also influence that 
of the mixture.

If the average of partial specific volume of DM \& NP-10 by dD/dc \& $\mathrm{V}_{\mathrm{F}}$ methods was used to calculate the partial specific volume of mixtures using equation 7 , the obtained value is 0.878 which is the same as the average from $\mathrm{dD} / \mathrm{dc} \& \mathrm{~V}_{\mathrm{F}}$ methods. This suggests that there is no interaction between these two molecules in mixed micelles. This finding supports the conclusion from surface tension measurements. The interaction parameter in mixed micelle is close to zero denoting no interaction. Since density measurements are fast and accurate, the traditional surface tension measurement may be replaced by partial specific volume determination to elucidate interactions between molecules in mixed micelles.

The partial specific volumes of DM from literature [8] has a $2.1 \%$ deviation in the case of experimental results and 3.3\% in the case of theoretical evaluation. Since DM used in the literature is not specified as ", \$ or mixtures of " and \$ conformations and the tested temperature is not given, the result is reasonable. The partial specific volume of Triton N-101 from literature [9] is used to compare with that of NP-10 and the deviation is only $0.3 \%$. Triton N-101's chemical formula is $\mathrm{C}_{9} \mathrm{NEO}_{9-10}$ while the NP-10's formula is $\mathrm{C}_{9} \mathrm{NEO}_{10}$.

\section{Temperature effect}

Temperature can have a marked effect on micellization, adsorption on rock and porous media and rheology of injected chemicals in the flooding process. It is our goal to investigate the change in micellar species and conformation.

The partial specific volumes of $1: 1$ mixtures at $25,30,36.8,40,45$ and $50^{\circ} \mathrm{C}$ are further determined to elucidate the effect of temperature and determine the temperature coefficient. Usually temperature coefficients of surfactants range from to $10 \times 10^{-4} \mathrm{~cm}^{3} \mathrm{~g}^{-1} \mathrm{~K}^{-1}$. The surfactant mixture may 
be a good sample for investigation partly due to the rigid two sugar rings connected by an oxygen atom and partly due to the mixing of n-dodecyl-\$-D-maltoside(DM) with nonyl phenol ethoxylated decyl ether (NP-10).

The temperature coefficient is defined as:

$$
f=\frac{d \bar{v}}{d t}
$$

$d t$
Here, $f$ is temperature coefficient, $\frac{d \bar{v}}{d t}$ is slope of partial specific volume versus temperature

Figure 5 shows the density of 1:1 mixtures at different temperature. Higher the temperature, the lower is the density. Although the logarithm plots suggests sharp increase at high concentrations, densities are actually linear with concentration.

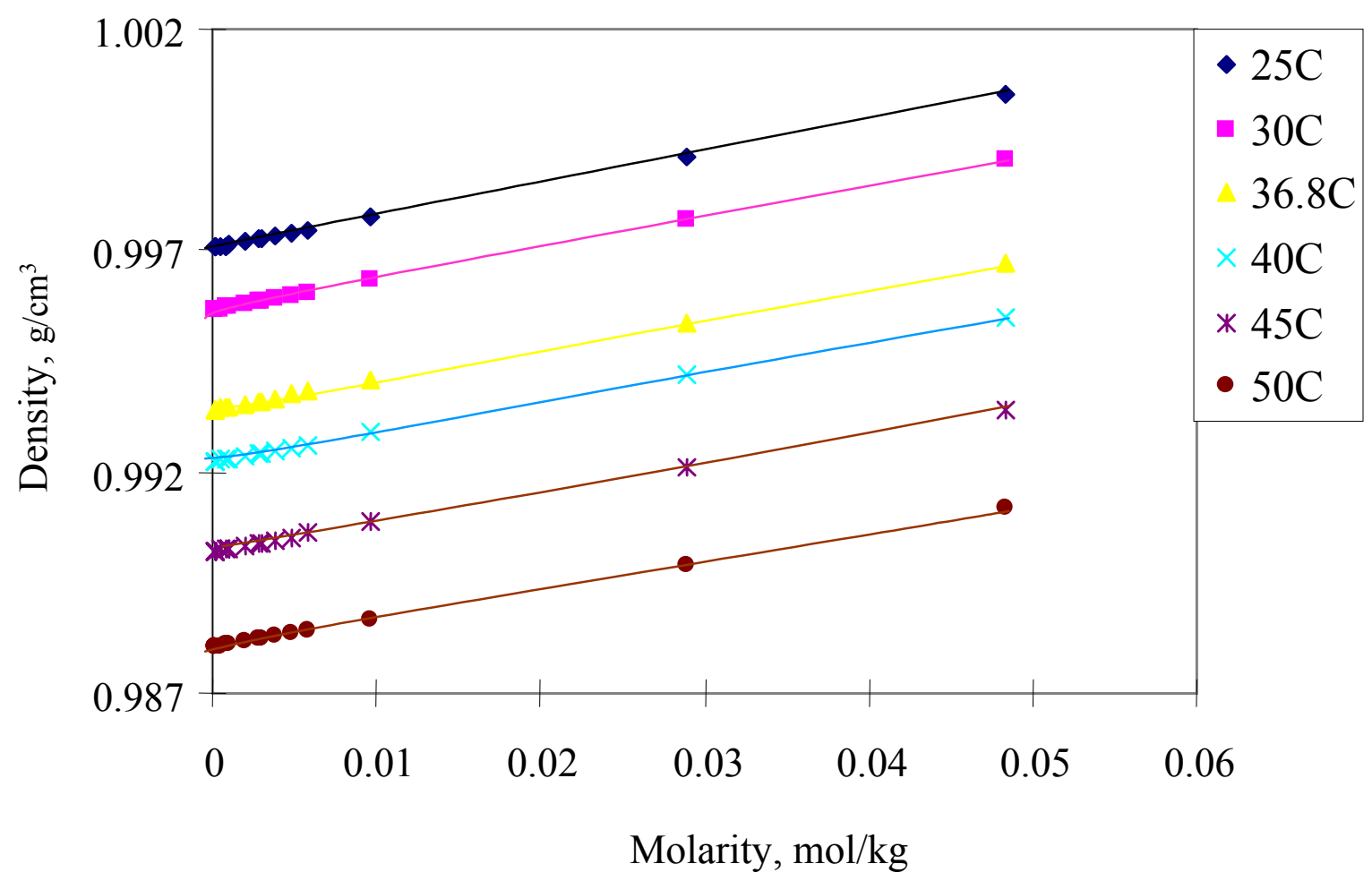

Figure 5. Density of 1:1 mixture of DM and NP-10 aqueous solutions at different temperatures

The data is analyzed with the above two methods: $d D d d c$ and $V_{F}$. dD/de method was used 
first. Table 6 contains the partial specific volumes of 1:1 mixtures at different concentrations and different temperatures. Here the $\mathrm{cmc}$ at each temperature was not measured. Thus the concentrations were divided to low, medium and high ranges. The low range consists of concentration of $10^{-5}$ to $10^{-}$ ${ }^{4} \mathrm{M}$ and medium range covers $10^{-4}$ to $10^{-3} \mathrm{M}$ while high range starts from $5 \times 10^{-3} \mathrm{M}$.

Table 6. the partial specific volumes analyzed for different concentrations and different temperatures by $\mathrm{d} D \mathrm{dc}$ method.

\begin{tabular}{|c|c|c|c|c|c|c|}
\hline \multirow{2}{*}{ Conc. Range } & \multicolumn{7}{|c|}{ Temperature, ${ }^{\circ} \mathrm{C}$} \\
\cline { 2 - 7 } & 25 & 30 & 36.8 & 40 & 45 & 50 \\
\hline low & 0.850 & 0.517 & & 0.730 & 0.735 & 0.778 \\
\hline medium & 0.879 & 0.884 & 0.888 & 0.892 & 0.895 & 0.898 \\
\hline high & 0.879 & 0.884 & 0.889 & 0.891 & 0.895 & 0.899 \\
\hline
\end{tabular}

The trend agrees with those obtained for DM, NP-10 and 1:1 mixtures at $25^{\circ} \mathrm{C}$. At the same temperature, the partial specific volume is smaller at low concentrations and higher at medium and high concentrations. Another trend is shown by the constant partial specific volume at medium and high concentration ranges. The partial specific volume increases with concentration.

When analyzed by $V_{F}$ method, the data for partial specific volumes are found to be scattered at low concentrations. The results are shown in figure 6.

From figure 6, it is obvious that the partial specific volumes jump at low concentrations and become constant afterwards. The partial specific volumes at concentrations higher than $0.002 \mathrm{~mol} / \mathrm{kg}$ are averaged and compared with the results obtained by $\mathrm{dD} d \mathrm{dc}$ method at high concentrations Table 7 and figure 7 show the values obtained. 


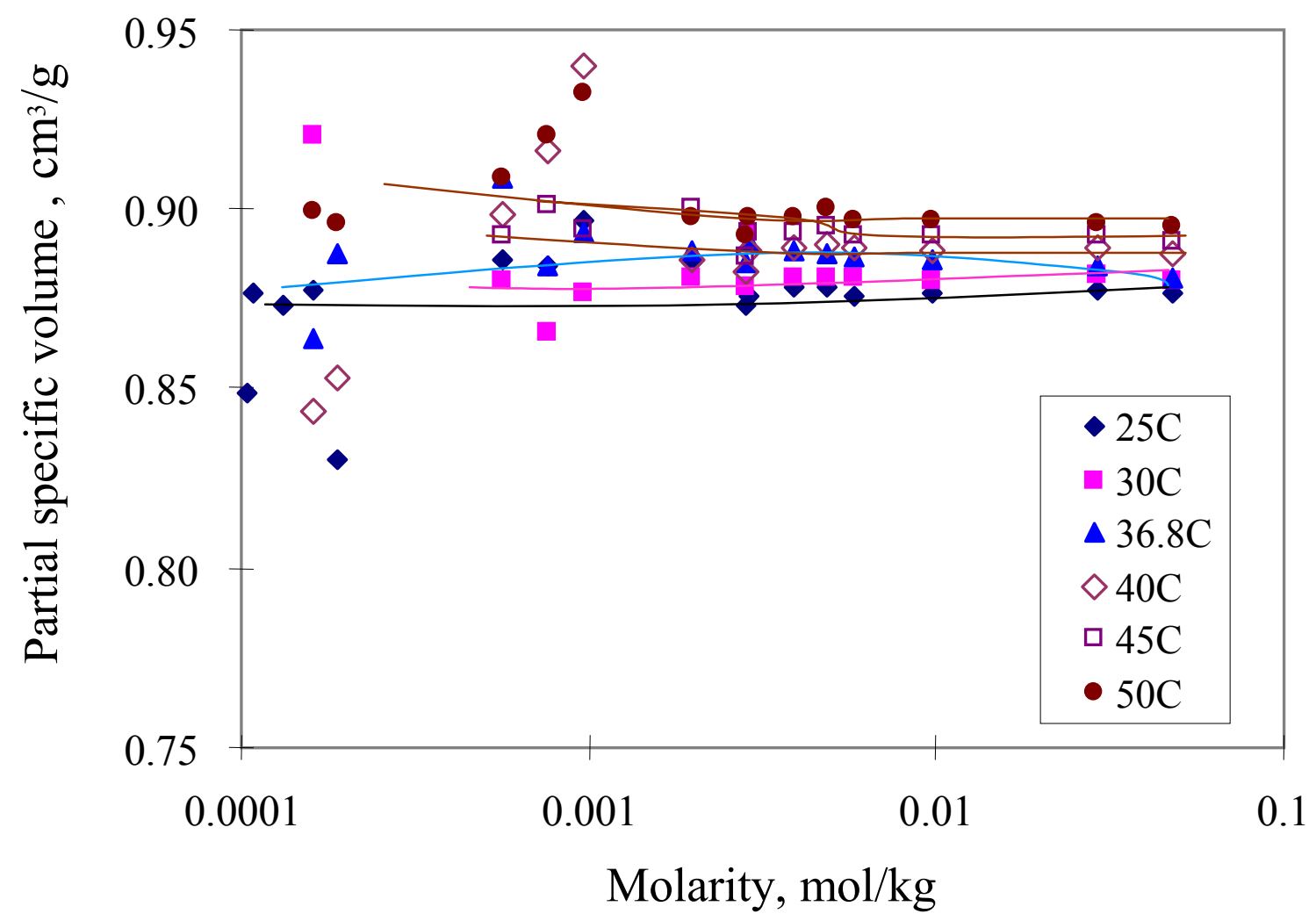

Figure 6. Partial specific volume of 1:1 mixture at different temperature versus molarity

Table 7. Partial specific volumes of $1: 1$ mixtures by $d D d c \& V_{F}$ methods with deviations.

\begin{tabular}{|c|c|c|c|c|c|c|c|}
\hline temp.,${ }^{\circ} \mathrm{C}$ & $\mathrm{dD} d \mathrm{dc}$ & $\mathrm{V}_{\mathrm{F}}$ & )$<\%$ & temp.,${ }^{\circ} \mathrm{C}$ & $\mathrm{dD} d \mathrm{dc}$ & $\mathrm{V}_{\mathrm{F}}$ & )$<\%$ \\
\hline 25 & 0.879 & 0.876 & 0.4 & 40 & 0.891 & 0.888 & 0.4 \\
\hline 30 & 0.884 & 0.880 & 0.5 & 45 & 0.895 & 0.892 & 0.3 \\
\hline 36.8 & 0.889 & 0.885 & 0.4 & 50 & 0.899 & 0.897 & 0.2 \\
\hline
\end{tabular}

The deviation between these two methods are within $0.2-0.5 \%$. From the $\mathrm{d} \bar{v} / \mathrm{d} t$, the temperature coefficients from both methods are obtained. Although there is small difference between the values by $d D d c \& V_{F}$ methods, the temperature coefficients are the same: $f=8 \times 10^{-4}$ $\mathrm{cm}^{3} \mathrm{~g}^{-1} \mathrm{~K}^{-1}$. 


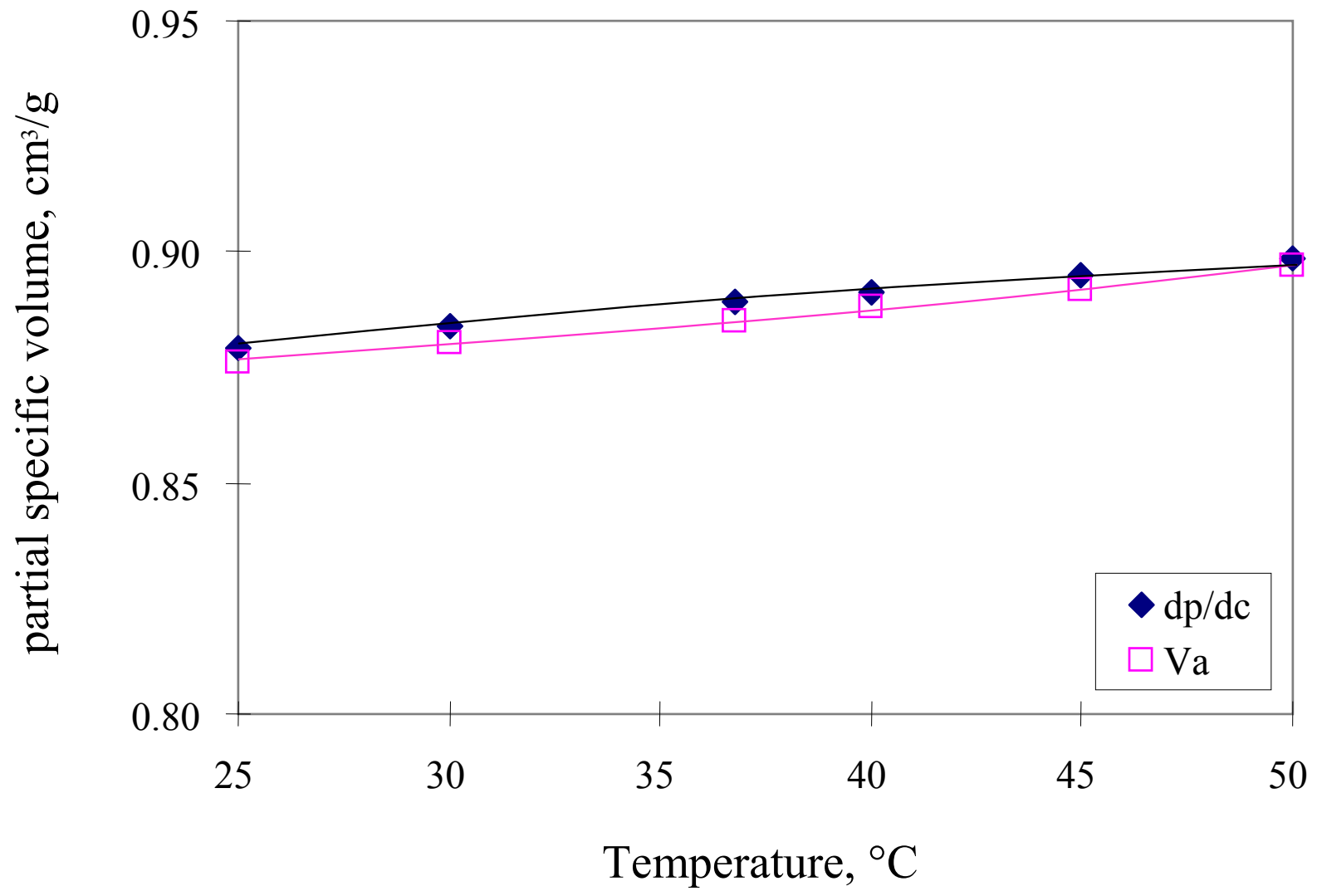

Figure 7. Partial specific volumes versus temperature by $d D d c \& V_{F}$ methods

The large temperature coefficient $\mathrm{f}$ indicates that the mixed DM and NP-10 micelles are sensitive to temperature gradients. In other words, mixed micelle has good fluidity at high temperatures which will facilitate the EOR process. 


\section{SUMMARY AND CONCLUSIONS}

Surface tension and density measurements have been conducted to determine critical

micellar concentrations (cmcs), partial specific volumes of n-dodecyl-\$-D-maltoside (DM), nonyl phenol ethoxylated decyl ether (NP-10) and their 1:1 mixtures at $25^{\circ} \mathrm{C}$. Durchschlag's theoretical method is also used to calculate partial specific volumes. The difference between different determination methods are investigated. Influence of temperature, mixing and micellization on partial specific volumes are as follows:

1. cmcs, maximum adsorption, area occupied per molecule and Gibbs free energy to form a micelle of n-dodecyl-\$-D-maltoside (DM), nonyl phenol ethoxylated decyl ether (NP-10) and their 1:1 mixtures are calculated. DM's parameters agrees with literature values well. There is no interaction between the two surfactant molecules in mixed micelles.

2. Partial specific volumes are obtained using two empirical methods: $\mathrm{dD} d \mathrm{dc}$ and $\mathrm{V}_{\mathrm{F}}$. The difference between data obtained using these two methods is within $0.5 \%$. However, Durchschlag's theoretical calculation method has a deviation of -3.3 to $-0.3 \%$ from experimental results. The rigid sugar group structure of DM is responsible for the large deviation. This reinforces the importance of packing of molecules in mixed micelles.

3. Micellization has a big effect on the partial specific volume. Below the $\mathrm{cmc}$, the value is smaller than that above cmc. Deviation of the partial specific volume are $0.4 \%, 1.9 \%$ and $2.3 \%$ for DM, mixture and NP-10, respectively, when concentration is below 10 times $\mathrm{cmc}$ Above that, the partial specific volumes are constant. The values are 0.820 for DM, 0.878 for 1:1 mixture and 0.921 for NP-10.

4. Mixing has no effect on partial specific volume. The results from the partial specific 
volumes suggest that there is no interaction between the single surfactant components in the mixed micelles.

5. The temperature coefficient is computed to be $8 \times 10^{-4} \mathrm{~cm}^{3} \mathrm{~g}^{-1} \mathrm{~K}^{-1}$ using both $\mathrm{dD}$ dc and $\mathrm{V}_{\mathrm{F}}$ methods ranging from $25-50^{\circ} \mathrm{C}$. This implies that the mixed micelle is sensitive to change in temperature. The partial specific volume at high temperatures is larger than that at low temperatures. Thus the fluidity of surfactants mixed solutions is enhanced under reservoir temperature conditions, requiring reduced pressure for injecting chemicals in chemical flooding process.

\section{FUTURE PLANS}

* Perform zeta-potential measurement of surfactant solutions adsorbed solids to examine the electrokinetic properties. This may explain the previous adsorption of mixed surfactants on solid surfaces from the surface charge point of view.

* Unlike other analytical software in the analytical untracentrifuge study, SEDFIT is able to analyze particles of smaller molecular weight such as micelles and distinguish species of slightly difference in molecular weights in solutions. Our proposed model shows that two types of surfactant mixed micelle do not have large differences in micellar mass. We plan to utilize SEDFIT to analyze the sedimentation velocity experiment data.

* Run sedimentation equilibrium experiment to obtain information on nonideality in surfactant mixtures and interaction of surfactants with polymers.

* Apply ultrafiltration technique to obtain percentage of each surfactant component in mixed micelles at different concentrations.

* Measure the diffusion coefficient, hydration and micellar weight by dynamic \& static light 
scattering and correlate the data from dynamic \& static light scattering with the results from analytical ultracentrifuge.

\section{PUBLICATION \& PRESENTATION}

1. R. Zhang, L. Zhang and P. Somasundaran. "Surface tension and fluorescence study of n-dodecyl-

\$-D-maltoside with anion, cationic and nonionic surfactant mixtures in aqueous solution”, to be submitted

2. A. D. Campbell, Z. Pan and P.Somasundaran. in V. Hackley, P.Somasundaran and J. A. Lewis, eds. "Polymers in Particular Systems - Properties and Applications", Marcel Dekker, Inc, New York, 2001

3. Q. Qiang and P. Somasundaran. Journal of Colloid and Interface Science, 253, 231-237, 2002 4. L. Zhang and P. Somasundaran, "Adsorption of Mixtures of Nonionic Sugar-based Surfactants" presented at the $76^{\text {th }}$ ACS Colloid \& Surface Science Symposium, June 23-26, 2002. Ann Arbor, Michigan. 


\section{REFERENCES}

1. Calum J. Drummond, Gregory G. Warr, Franz Grieser, Barry W. Ninham and D. Fennell Evans, "Surface properties and micellar interfacial micrenvironment of n-dodecyl-\$-D-maltoside", J. Phys. Chem. B. 1985, 89, 2103-2109

2.J. A. Molina-Bolívar, J. Aguiar and C. Carnero Ruiz, "Growth and hydration of Triton X-100 micelles in monovalent alkali salts: a light scattering study", Molecular Physics, 2001, Vol. 99, No. 20, 1729-1741

3. A. González-Pérez, J. L. Del Castillo, J. Czapkiewicz, and J. R. Rodríguez, “Temperature dependence of equilibrium and transport properties of decyldimethylbenzylammonium chloride in aqueous solutions", J. Chem. Eng. Data 2001, 46, 709-711

4. C. Dupuy, X. Auvray, and C. Petipas, "Anomeric effects on the structure of micelles of alkyl maltosides in water" langmuir 1997, 13, 3965-3967.

5. K. S. Birdi, "The size, shape and hydration of micelles in aqueous medium", Progress in colloid \& polymer science, $1985,70,23-29$.

6. H. Durchschlag and P. Zipper, "Calculation of the partial specific volume of organic compounds and polymers", Progress in Colloid \& Polymer Science, 1994, 94, 20-39

7. Helmut Durchschlag and Peter Zipper, "Calculation of partial specific volumes of detergents and lipids”, Jorn. Com. Esp. Deterg., 1995, 26, 275-292

8. Timmins, P. A., Leonhard, M., Weltzien, H.U., Wacker, T., Welte, W., "A physical characterization of some detergents of potential use for membrane protein crystallization", FEBS Lett. 238, 361-368, 1988

9. Steele, J. C. H. Jr., Tanford, C., Reynolds, J. A., "Determination of partial specific volumes for lipid associated proteins", Methods Enzymol. 48 (1978), 11-23 